\title{
Local Coordination in Metal-Organic Frameworks Probed in the Vibrational and Optical Regime by EELS
}

\author{
Sean M. Collins ${ }^{1 *}$, Demie M. Kepaptsoglou², Keith Butler ${ }^{3}$, Louis Longley ${ }^{1}$, Jingwei Hou ${ }^{1}$, Thomas D. \\ Bennett $^{1}$, Quentin Ramasse ${ }^{4}$ and Paul Midgley ${ }^{1}$ \\ 1. Department of Materials Science and Metallurgy, University of Cambridge, Cambridge, UK. \\ 2. SuperSTEM, Daresbury, UK and Department of Physics, University of York, York, UK. \\ 3. ISIS Facility, Rutherford Appleton Laboratory, Harwell Oxford, Didcot, UK. \\ 4. SuperSTEM, Daresbury, UK and School of Chemical and Process Engineering and School of Physics, \\ University of Leeds, Leeds, UK. \\ * Corresponding author: smc204@cam.ac.uk
}

Metal-organic frameworks (MOFs) encompass a broad family of materials built from transition metal ions and organic linker molecules. In their crystalline form, some MOFs exhibit high porosities and show significant promise in gas storage applications. A number of MOFs, most notably members of the zeolitic imidazolate framework (ZIF) family, can also be prepared as melt-quenched glasses which retain the local metal-ligand coordination [1]. MOF glasses offer favorable processability and exhibit tunable mechanical properties intermediate to those of the previously known organic, inorganic, and metallic glasses [2]. Many MOF applications require MOF composite materials with multifunctional properties, motivating microscopic characterization of the local electronic structure by electron beam techniques.

The effects on the electronic structure arising from the crystal to glass phase transition or liquid-phase processing of multicomponent MOFs is not well studied, prompting the development of microanalytical techniques for determination of the local coordination chemistry. Metal-organic materials are, however, prone to structural collapse or decomposition under electron beam irradiation. These characteristics present particular challenges for spectroscopic measurements in electron microscopy. Here, by combining monochromated electron energy loss spectroscopy (EELS) at high energy resolution with machine learning techniques, low signal-to-noise spectra are processed to map spatially varying optical and vibrational electronic structure of multicomponent MOF materials.

First, a glass blend comprised of a Zn phase (ZIF-62) and a Co phase (ZIF-4-Co) was examined at visible and ultraviolet energies [3]. For ZIFs, local tetrahedral coordination of the metal center is observed in both crystalline and glass phases, in direct analogy to silica. Tetrahedral Co exhibits $d$ - $d$ transitions at visible energies, and ZIF-4-Co is known to exhibit promising photocatalytic properties [4]. To examine how these optical properties are preserved in a microstructured glass blend, monochromated low loss EELS and correlated core loss EELS at the metal center $L_{23}$ edges were carried out using a $\operatorname{Titan}^{3}$ 80-300 microscope (Thermo Fisher), operated at $80 \mathrm{kV}$ and $300 \mathrm{kV}$ with a recorded energy resolution of $<180 \mathrm{meV}$ (full width at half maximum of the zero loss peak), as well as a Hermes UltraSTEM 100MC (Nion), operated at $60 \mathrm{kV}$ with an energy resolution of approximately $25 \mathrm{meV}$.

Spectra were recorded from precursor crystalline ZIF phases as well as from the glass blend. The crystalline precursors and glasses exhibited indistinguishable spectra. A peak at $2 \mathrm{eV}$ was observed only for Co phases, and additional differences were also observed between 4-10 eV (Figure 1). Non-negative matrix factorization (NMF) was used to form maps of EELS spectra recorded at high noise levels to 
minimize beam damage. The NMF algorithm extracted spectra with features matching those observed in selected area spectra, including the $2 \mathrm{eV}$ peak indicative of $d$ - $d$ transitions in the ZIF-4-Co phase. These maps were experimentally corroborated by core loss EELS, and the domains found by NMF of low loss spectra were identical to those observed in core loss EELS maps. The peak assignments were further validated using density functional theory (DFT) calculations of the loss function [3].

In multicomponent MOFs containing two different linker functional groups, vibrational EELS provides additional insight into the ligand domain structure, crucially measured using a signal independent from those used to map the metal center domains. In this second case, a crystal-glass composite made up of a glass matrix (ZIF-62) containing particles of crystalline MIL-53 [5] was analyzed by monochromated EELS as well as by correlated X-ray energy dispersive spectroscopy (EDS). The ZIF-62 phase contains Zn metal centers and imidazolate ligands $\left(\mathrm{C}_{3} \mathrm{H}_{3} \mathrm{~N}_{2}{ }^{-}\right)$whereas MIL-53 incorporates a $\mathrm{Al}$ metal centers and benzene dicarboxylate $\left(\left[\mathrm{O}_{2} \mathrm{C}-\mathrm{C}_{6} \mathrm{H}_{4}-\mathrm{CO}_{2}\right]^{2-}\right)$ linkers. NMF extracted distinct signatures from vibrational EELS which verified ligand functional groups remain coordinated within the corresponding metal center domains. These findings were corroborated by elemental mapping and by the fine structure analysis at the $\mathrm{C} K$ edge. The vibrational EELS maps exhibited a spatial resolution estimated at $<15 \mathrm{~nm}(80 \%$ criterion).

Both the ligand field splitting of the metal center and the ligand functional groups have been mapped with EELS at nanometer spatial resolution. Cumulatively, these findings establish low loss EELS as a tool for mapping local coordination chemistry in metal-organic solids [6].

\section{References:}

[1] T. Bennett et al. Journal of the American Chemical Society 138 (2016), p. 3484.

[2] L. Longley et al. Nature Communications 9 (2018), p. 2135.

[3] S.M. Collins et al. Journal of the American Chemical Society 140 (2018), p. 17862.

[4] B. Pattengale et al. Journal of the American Chemical Society 138 (2016), p. 8072.

[5] J. Hou et al. ChemRxiv. DOI: 10.26434/chemrxiv.7093862.

[6] S.M.C. gratefully acknowledges support from the Henslow Research Fellowship at Girton College, Cambridge. P.A.M. acknowledges support from the EPSRC (EP/R025517). SuperSTEM is the UK National Research Facility for Advanced Electron Microscopy, supported by EPSRC. The research was also supported by the European Research Council (291522-3DIMAGE).
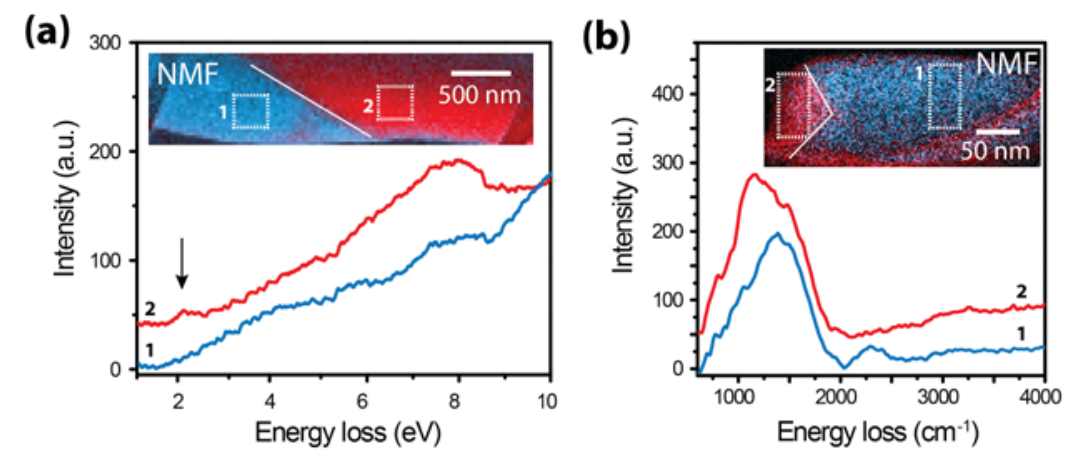

Figure 1. Background subtracted selected area spectra and (inset) NMF maps for (a) a glass blend formed from ZIF-62 (1, left, blue) and ZIF-4-Co (2, right, red) and (b) a crystal-glass composite particle formed from ZIF-62 (1, right, blue) and MIL-53 (2, left, red). Selected areas are numbered and marked with by dashes squares and the interfaces are marked by solid lines on the NMF maps. 